Review article

Paediatrics Today 2015;11(2):93-114

DOI $10.5457 /$ p2005-114.116

\title{
EARLY PSYCHOLOGICAL DEVELOPMENT OF VERY PRETERM AND/OR LOW BIRTH WEIGHT CHILDREN: A REVIEW OF THE LITERATURE
}

\author{
Maria-José GONZÁLEZ-VALENZUELA*, Milagros FERNÁNDEZ-MOLINA, \\ Myriam DELGADO-RÍOS
}

Department of Development and Educational Psychology, Faculty of Psychology, University of Málaga Málaga, Spain

\footnotetext{
${ }^{*}$ Corresponding author: valenzu@uma.es

Tel.: + 34952132484

Fax.: + 34952132635
}

Received: November 25, 2014

Accepted: March 30, 2015

Key words: Premature infant Child development - Language Cognition • Emotion.

\begin{abstract}
Objective - The aim of this paper is to describe the psychological development in three areas (cognitive, communicative-linguistic and social-emotional domains) of very preterm children and/or low birth weight, aged from 0 to 6 years, to discover if problems exist and if these difficulties remain, decrease or increase over time, up to 6 years of age, through of review of studies done in this population. Materials and methods - Seven databases were searched. The search yielded 975 studies and those related to cognitive, communicative-linguistic and social-emotional development in children without physical problems, ranging in age from birth to 6 years, gestational age less than 32 weeks and/or birth-weight less than 2499 grams were analyzed. Most of the 47 studies analyzed were conducted after 2005, focused on children aged 3-5 years, and compared preterm and full-term infants. Results The studies agree that preterm children differ in many developmental domains but there is no consensus on others. Several studies found a close association between the three different areas analyzed. Conclusion - Early intervention of preterm and/or low birth weight children is the most important way to prevent and/or reduce academic, communicative and social-emotional (anxiety/depression) problems, and improve long-term adaptation to the environment, self-regulation, communication skills, social competence and academic performance in general.
\end{abstract}

\section{Introduction}

The World Health Organization classifies those born before week 28 of gestation as extremely preterm, those born between weeks 28 and 32 as very preterm, and those born between weeks 32 and 37 moderate or late preterm (1). According to the ICD-10 classification, when the variables "birth weight" and "gestational age" are both available, priority of assignment should be given to birth weight, excluding low weight due to slow fetal growth and fetal malnutrition. In the category of disorders related to length of gestation and fetal growth (P05-P08), ICD-10 distinguishes between extremely low birth weight (ELBW; <999 grams), other low birth weight (1000-2499 grams), extreme immaturity ( $<28$ weeks of gestation), and other preterm children (between 28 and 37 weeks gestation) (2). Thus, preterm birth occurs before 37 weeks of gestation, and is considered a biological and psychological risk factor for the increased probability of neurological, intellectual and adaptation problems following 
birth $(3,4)$. Thus, preterm children are a heterogeneous group because their prenatal development, birth gestational week or weight, and postnatal medical problems, can be very different $(5,6)$. Historically, it was thought that preterm children were a homogeneous group with little chance of survival, because if they overcame the more severe medical complications associated with being preterm; they would be more likely to develop disorders of all types later in life, independently of other factors $(1,7,8,9,10)$.

Preterm children are of great scientific interest regarding development course, mainly sensory-motor and physical development $(11,12,13,14,15,16)$, and the effect on child development of prenatal variables (tobacco, stress, drugs, infections, etc.), perinatal variables (breathing problems, brain damage, environment, and family care, etc.) (17, 5, 6). Developmental domains of interest are physical sensory-motor development (sleep or food problems, motor reflex, visual-motor or perceptual-motor abilities, etc.), cognitive development (perception, attention, memory, IQ, cognitive skills, executive functionEF-, etc.), language development (vocabulary size, phonological memory, comprehension, production, grammatical rules, speech sound discrimination, naming, etc.), and socialemotional development (play interaction, social competence and social skills, behavior problems, temperament, mother-child attachment, emotional regulation, emotional well-being, etc.).

Since the 1990s, improvements in perinatal and neonatal care units have led to increased survival rates (from 5\% to 10\%) even when the gestational age at birth is younger $(18,19)$. Thus, in the last decade there has been a striking increase in investigations into how birth weight and gestational age influence psycho-neurological development. For example, depending on the gestational age, it is known that between $35 \%$ and $40 \%$ of preterm children require special care for 5 years. There is also increased interest in the potential psychological effects in this population. Relevant interest teams or networks have been created in hospitals that may be linked to universities, and these teams have analyzed large samples of VLBW and ELBW infants; for example, the NICHD or $\mathrm{Na}$ tional Institute of Child Health and Human Development Neonatal Research Study (20), the VON or Vermont Oxford Network (8, 9), the EPICure study (10) or the EPIPAGE (21).

Currently, neonatologists and pediatricians are looking for information on the consequences of prematurity on child psychological development so that they can make informed clinical decisions $(10,21,20)$. The interest of prenatal counseling in cases of potential iatrogenic preterm birth or fetal clinical conditions, should be also focused on this. Thus, a research area of interest would be to identify specific areas of child psychological development that should be further analyzed during the early period onward, to detect potential precursors of maladaptation in each child and the likelihood of later problems in their cognitive, communicative, emotional, and social behavior. Therefore, this population is studied due to the importance of understanding the psychological development of these subjects, since in recent years their survival rate has been high and they need early intervention, when compared amongst themselves and with their peers (1).

However, on the other hand, efforts to collect and systematize the most relevant results of the latest scientific research on preterm children - that is, systematic review studies and meta-analyses - have focused on just one or two developmental and psychological domains rather than all of them, such as the psychomotor area $(11,12,13,14,15$, 16). For example, some of the developmental domains investigated separately are: lan- 
guage functions (22), EF and attention (4), academic achievement, EF and behavioral problems (3), handedness (23), psychiatric diagnoses (24), infant-mother attachment (25), quality of life (26), and cognitive and behavioral outcomes (27). Besides this, the researchers focus on the area of motor development because psychomotor difficulties are more obvious than others from birth, and parents or pediatricians are more concerned about this domain. Some studies have also analyzed preterms when they are in adolescence or adulthood, although early detection is of little relevance in these groups and thus they are less likely to achieve a normal developmental trajectory. Thus, there is a lack of studies that include several of these developmental domains together or that focus on the early stage (0-6 years), which is precisely the most sensitive period for learning and child development.

Thus, the aim of this paper is to describe psychological development in three areas (cognitive, communicative-linguistic and social-emotional domains) of very preterm children and/or low birth weight children, aged from 0 to 6 years, to discover if there are problems and if these difficulties remain, decrease or increase over time, up to 6 years, through of review studies done in this population.

\section{Methods}

\section{Search strategy}

The databases ERIC, ProQuest Psychology Journals, PsycArticles, Psycritiques, Psycinfo, Medline and ProQuest Health \& Medical Complete were searched for articles. Also, reference lists of published articles were reviewed to identify further relevant research.

The search used terms and strings such as: preterm-born children + preschool + "language development"; preterm-born children + preschool + "cognition development"; and preterm-born children + preschool + "emotional development".

The criteria used to search for target studies were articles published in English in peer-reviewed scientific journals between 1990 and 2012. These criteria were used in previous studies in reviews in preterm children $(24,28,22)$. The articles found were screened by title and by abstract.

This is a scoping review in that it attempts to unify different texts around a common theme, i.e., the psychological development of preterm children during early childhood. It is not an integrative or interpretative review, since no meta-analysis or interpretation of the theoretical perspective were conducted, respectively (29).

\section{Study selection}

Fig. 1 shows a flow chart of the study selection process, according to PRISMA 2009 Flow Diagram (30). The search yielded a total of 975 studies, through database researching and reference lists of published articles. 676 articles were discarded, based on the screening of titles and abstracts, according to the research strategy. 299 full-text articles were analyzed to determine their eligibility. 252 full-text articles were excluded, according to the different exclusion criteria. These criteria were the following: review articles, commentaries and editorials, because these are not experimental studies; studies with participants with organic problems (sensory and motor handicaps, congenital abnormalities, neurological problems), because these handicaps could have a negative influence on the outcomes; longitudinal studies that started before the participants were 6 years old and ended after they were more than 6 years old; those born at a gestational age more than 32 weeks (according to The World Health Organization classifies) and/or birth weight greater than 2499 grams (according to ICD-10), 
according to the study aims. Some examples of these excluded articles were the studies by Caravale et al. (31), the study by Esbjorn et al. (32), and the study by Delobel-Ayoub et al. (33).

In total, 47 studies were selected for review. The articles selected were studies published in English in peer-reviewed scientific journals between 1990 and 2012 (not review articles and commentaries and editorials) about cognitive development, communicative-linguistic development and social-emotional development, with participants less than 6 years old whom they did not begin to evaluate before 6 years of age and which ended after this age, without organic problems and a gestational age less than 32 weeks, and/or birth weight less than 2499 grams.

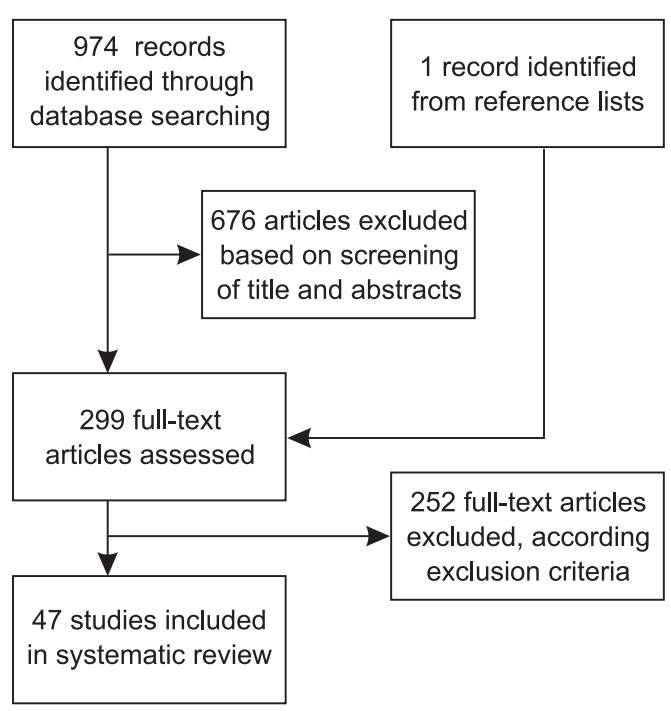

Fig. 1 Flowchart of the different stages of the review.

\section{Data extraction}

First, one of the authors conducted the general search. Second, three authors analyzed the titles and abstracts in order to exclude articles according to the general criteria. Third, three authors analyzed full-text articles for eligibility and excluded full-text articles ac- cording to the exclusion criteria. The studies chosen for analysis by consensus were classified according to these areas of psychological development: cognitive, communicative-linguistic and social-emotional. After this, each author analyzed the studies included in the review for one of these areas. If any articles contained information on different areas, these studies were examined by the authors responsible for reviewing studies on those areas. Thus, after the selected studies were analyzed, the following information was extracted from each study for their authors: reference and year of publication, country, age range or mean, study design (comparative, longitudinal, follow up study, pre-test-posttest), control group characteristics (number, gestational age, birth weight), study group characteristics (number, gestational age, birth weight) and variable type. The instruments used in the study were also analyzed and discussed in the text. Finally, analyzed studies were organized by year of publication, with the aim of viewing the obtained results in cognitive development, communicativelinguistic development and social-emotional development.

\section{Results}

Of the studies reviewed, six focused on cognitive development, 14 on communicativelinguistic development, and 13 on socialemotional development. Six studies analyzed aspects of both cognitive and communicative-linguistic development, seven analyzed aspects of cognitive and social-emotional development, and just one investigated all three aspects of psychological development. Specifically, of the total number of studies analyzed, $42.55 \%$ were related to cognitive development, $44.68 \%$ to communicativelinguistic development and $44.68 \%$ to social-emotional development (Tables 1, 2, 3).

Regarding studies on the psychological development of preterm children, $66 \%$ were 
published since 2005 , were conducted in different countries, and varied according to the area of psychological development studied. Six of the studies on cognitive development that we analyzed were conducted in the USA, four in the Netherlands, three in Italy, two in the UK and the rest in various countries. The numbers of those carried out on communicative-linguistic development were: first five in the UK and four in Finland, followed by two in Italy, two in the USA, Australia and the Netherlands, and the rest in various countries. Finally, seven of those on social-emotional development were conducted in the USA, five in Australia, two in Finland and the rest in various countries (Tables 1, 2, 3).

Thus, nine studies on cognitive development focused on preterm children between $2-4$ years old, six before 1 year of age and five between 5 and 6 years of age. Fourteen studies on communicative-linguistic development focused on preterm children between 2 and 5 years of age, seven studies between 5 and 6 years of age and one study before one year of age. Ten studies on social-emotional development focused on preterm children before one year of age, eight studies between 2 and 5 years of age and two studies between 5 and 6 years of age (Tables 1, 2, 3).

The comparative study design was found in 10 studies on cognitive and social-emotional development, respectively, and in 15 studies on communicative-linguistic development. The longitudinal study design was found in four studies on cognitive development and in one on communicative-linguistic development. The longitudinal and comparative study design was found in seven studied on social-emotional development, in four on cognitive development and in one on communicative-linguistic development. The follow up study design was found in three studies on communicative-linguistic development, in two on cognitive development and in one on social-emotional development.
The pre-test-post-test study design was only found in three studies on social-emotional development. There was an experimental group but no control group in just 10 of the studies analyzed (Tables 1, 2, 3).

In the sample study group on cognitive development 11 of the studies consisted of 30-100 participants and four studies of less than 30 or more than 100 participants, respectively. Eight studies on communicativelinguistic development had a study group sample with more than 100 participants, seven studies with less than 30 participants in the study group and six studies had between 30-100 participants in this group. Finally, 11 of those on social-emotional development had a sample of between 30-100 participants in the study group, seven studies had more than 100 participants and two studies with less than 30 participants.

The gestational age of participants in the group study in the 11 studies on cognitive development was $<30-32$ weeks, in seven studies $<36$ weeks and in two studies $<28$ weeks. In the studies on communicative-linguistic development, the participants of the group study were born at $<30-32$ weeks in 11 studies, $<28$ weeks in 28 studies and $<36$ weeks in two studies. The gestational age of participants in the group study in 12 studies on social-emotional development was $<30$ 32 weeks, in seven studies $<36$ weeks and in one study $<28$ weeks (Tables $1,2,3$ ).

The birth weight of participants in the group studies in five studies on cognitive development was between 1000-2499 grams, in three studies < 1000 grams, in 3 studies $<1250$ grams and in four studies $<1750$ grams. In the studies on communicativelinguistic development, the participants in the group study weighed between 10002499 grams in seven studies, $<1000$ grams in one study, $<1250$ grams in four studies and $<1500$ grams in six studies. The birth weight of participants in the group study in five 
studies on social-emotional development was between 1000-2499 grams, in two studies $<1000$ grams, in three studies $<1250$ grams, in three studies $<1750$ grams and in three studies $<2500$ grams (Tables 1, 2, 3).

The main study variables in the studies on cognitive development were memory and attention, followed by general cognitive and executive function, while processing speed and perception were the least studied. In studies on communicative-linguistic development, the most used variables were expressive and receptive language, vocabulary, followed by general verbal skills, auditory-phonological abilities, naming objects and words, grammar and morphosyntax. In the area of socioemotional development, the variables most frequently used in the studies were parental behavior, emotional regulation and functioning, mother-child interaction, adaptive behavior problems, social competence and temperament (Tables 1, 2, 3).

Finally, the most frequently used instruments used to assess the cognitive ability of children in these studies were as follows: the Wechsler Preschool and Primary Scale of Intelligence-Revised (WPPSI-R), the Revisie Amsterdamse Kinder Intelligentie Test (RAKIT), the Bayley Scales of Infant Development, the McCarthy Scales for Children's Abilities (MSCA), Behavior Rating Inventory of Executive Function Preschool Version (BRIEF), the Leiter International Performance Scale-Revised (LIPS-R), the Shape School, Differential Ability Scale-Second edition (DAS-II), Neuropsychological Assessment (NEPSY) and the Rivermead Behavioral Memory Test (RBMT). A great variety of psychometric instruments were used to assess the communicative-linguistic development of children, according to the characteristics of the study variables. Among these, the most frequently used were tests from the McCarthy Scale (MSCA), some of the WISC and WPPSI tests, the Peabody test, the NEPSY neuropsychological battery, and the Reynell Scale. Other more specific tests were also found: the British Picture Vocabulary Scaleslong Form (BPVS), the Bus Story Test of Continuous Speech, Children's Test of Nonword Repetition, the Clinical Evaluation of Language Fundamentals (CELP-4), the Diagnostic Evaluation of Articulation and Phonology (DEAP), among others. Studies on social-emotional development used observational measures such as those involving the child carrying out daily activities and/or free play and/or structured tasks, carried out during mother-child social interaction: strange situation reunion, mother-child negotiation in free play, toy-centered play interaction, semi-structured tasks with different stimuli, such as puzzle board, posting box puzzle, and a clear plastic box with a lever inside, etc. On the other hand, the most frequently used standardized instruments in this area of study are: the Infant Toddler Social and Emotional Assessment (ITSEA), Subscales from the Vineland Adaptive Behavior Scale, Early Infancy Temperament Questionnaire (EITQ), Emotion Regulation Checklist, NEPSY, Temperament Italian Questionnaire (QUIT) and the Short Temperament Scale for Infants, among others.

The results obtained in the articles analyzed about each area of psychological development are discussed. Descriptive data on psychological development in the selected studies were classified according to the different areas analyzed: cognitive, communicative-linguistic and social-emotional. Within each domain, the data is presented according to age (from 0 to 6 years).

\section{Cognitive development studies}

Rose et al. (34) found that the speed of information processing is slower in preterm children during the first year of life. Similarly, Hugues et al. (35), found that at 12 
months of age preterm children differ in attention scores compared to full-term infants. Feldman (36) also found that these children have poorer results in sustained attention at 12 months. However, regarding executive functions, Evrard et al. (37) found no significant differences between very preterm children and full-term infants at both 12 months and 24 months of age. On the other hand, Hunnius et al. (38), found that until about 16 weeks post-term, preterm children were faster than full-term infants in disengaging and shifting their attention and gaze from a stimulus in their central visual field to the periphery. They also showed that visual and sensorimotor processing was more efficient in preterm children, rather than their having advanced attentional development (38).

Landry et al. (39) found that cognitive development was slower in preterm children during the first 2 years of life. On the other hand, Pitchford et al. (40), found significant differences in the development of attention and memory in preterm children at 2 years of age. They found that preterm boys are more likely to have cognitive problems than preterm girls (40).

Espy et al. (41) found deficits in emergent mathematics and the control and maintenance of attention in preterm children aged 3-4 years old. These deficits in cognition in preterm children are commonly found in association with abnormalities in blood $\mathrm{pH}$. Baron et al. (42) found significant differences in memory and spatial learning between low birth-weight children and late preterm and full-term infants. Vicari et al. (43) also compared memory, attention and spatial perception in low birth-weight children and fullterm children. The results show that preterm children may have specific difficulties in attention, visuospatial processing and working memory (43). In another study, Clark et al. (44) found that children at this age do not differ when moving from one task to another, but they differ in levels of sustained attention during problem solving. Furthermore, Dall'oglio et al. (45) found that these children had decreased neuropsychological performance in cognitive development, shortterm memory, visual motor skills and spatial construction, but not in visual processing. Woodward et al. (46) also found that these children had poorer performance in cognitive planning skills, selective attention and inhibitory control than full-term children, a finding that is supported by other studies that have found a close association between damage to white matter and deficits in executive function $(7,46)$.

Briscoe et al. (47) investigated 5-year-old preterm children and found that they were at increased risk of problems in everyday memory and cognitive impairment, particularly in the early years of schooling. These authors suggest a possible link between cognitive impairment and memory problems. Similarly, Roberts et al. (48) found that preterm children at 5 years of age had more problems in memory and executive function, which were closely associated with subsequent academic difficulties. They also had lower scores than their full-term peers in learning styles and cognitive abilities. Perricone and Morales (49) found difficulties in similar children in areas related to attention and motor control. They found a minor trend toward guiding and regulating their own attention, keeping focused on an object, and decreased capability of moving their attention from one stimulus to another. These cognitive difficulties were associated with difficulties in general intelligence rather than with specific dysfunctions (49). However, Soondar et al. (50) did not find significant differences in intelligence scores between preterm children and full-term children or in cognitive development, where $92 \%$ of the children tested were normal. Nordhov et al. (51) conducted a longitudinal study on preterm children of 
this age, assessing the effectiveness of early intervention on their cognitive development. They found that the children who underwent intervention obtained better scores on cognitive development than the control group.

Finally, Cornelieke et al. (52) conducted a study with very preterm children at 6 years of age, finding that these children had impaired development in executive function, and that these differences were independent of IQ. They also found that these children obtained low scores for executive function, associated with lower scores in working memory and concept generation tasks (52).

\section{Communicative-linguistic development studies}

Compared to full-term children, it has been found that in the first few days from birth, preterm children show atypical neural activity patterns during auditory discrimination activities and recognition of sounds, and also do not recognize their mother's voice (53).

Preterm children between 1 and 2 years of age also have lower scores than full-term children in communicative-linguistic skills and language development. Specifically, preterm children have lower scores than full-term children in the production of communicative gestures and word comprehension and production (54).

At 2 years, preterm children have impaired comprehensive and expressive language, independent of social risk, and lower scores in listening comprehension and expressive communication $(40,48)$. They use shorter sentences than full-term children (55) and have limited vocabulary and poor grammar skills in the use of predicates, verbs and adjectives, although they play more games and produce more animal noise words than full-term children (56). Similarly, Foster-Cohen et al. (57) found differences between preterm children of different gestational ages and full-term children, in vocabulary size and morphological and syntactic skills. Specifically, children with lower gestational ages have poorer language skills (vocabulary size and grammatical complexity), which persist even after controlling factors specific to the child (sex and birth weight) and to the family (ethnicity, type of family, socioeconomic status, mother's age at birth and family size). However, Marston et al. (58) did not find an association between vocabulary and gestational age and socioeconomic status. Stolt et al. (59) also found that there were no significant differences between preterm and full-term children at this age in vocabulary size (number of words produced), although they found that full-term children performed better in grammar (pronouns, prepositions, etc.) and the types of nouns used (social, common, etc.). Howard et al. (17) found that lower maternal education and poorer communication skills in preterm children at 2 years of age are strong predictors of expressive and receptive language problems at 5 years of age.

Briscoe et al. (60) found differences between preterm and full-term children at 3 years in receptive vocabulary and some components of expressive language (information), but did not find differences in other components of expressive language (sentences), non-verbal communication skills, and productive vocabulary. Espy et al. (41) also found that at this age preterm children experience problems with vocabulary. Van $\mathrm{Li}$ erde et al. (61) found that at this age preterm children had poorer receptive language skills (comprehension of questions, use of passive sentences, inference skills and use of prepositions), expressive language skills (defining words and expressing semantic relations) and morphosyntactic skills.

Four-year-old preterm children obtain poorer results in object naming and auditory discrimination tasks (55) and also obtain lower scores in verbal fluency and verbal compre- 
Table 1 Cognitive development

\begin{tabular}{|c|c|c|c|c|c|c|c|c|c|}
\hline \multirow{3}{*}{$\begin{array}{l}\text { Source and } \\
\text { country* }\end{array}$} & \multirow{3}{*}{$\begin{array}{l}\text { Age range } \\
\text { or mean }\end{array}$} & \multirow{3}{*}{$\begin{array}{l}\text { Study } \\
\text { design }\end{array}$} & \multicolumn{6}{|c|}{ Characteristic } & \multirow{3}{*}{ Cognitive variables } \\
\hline & & & \multicolumn{3}{|c|}{ Study group } & \multicolumn{3}{|c|}{ Control group } & \\
\hline & & & $\mathrm{N}$ & GA & BW & $\mathrm{N}$ & GA & BW & \\
\hline $\begin{array}{l}\text { Landry et al. } \\
\text { (1998), US }\end{array}$ & $\begin{array}{l}6,12,24 \\
36 \text { mos. }\end{array}$ & $\mathrm{L}$ & $\begin{array}{l}37 \\
42\end{array}$ & $<36$ & $<1600$ & 49 & $>36$ & NA & Cognitive development \\
\hline $\begin{array}{l}\text { Landry et al. } \\
(2000) \text {, US }\end{array}$ & $\begin{array}{l}6,12,24 \text { mos., } \\
3 \text { yrs. \& } 6 \text { mos., } \\
4 \text { yrs. \& } 6 \text { mos. }\end{array}$ & $C \& L$ & 73 & $<36$ & $<1600$ & 114 & $37-42$ & NA & $\begin{array}{l}\text { Cognitive skills; Maternal behaviors: } \\
\text { maintaining and directiveness, and } \\
\text { warm sensitivity }\end{array}$ \\
\hline $\begin{array}{l}\text { Briscoe et al. } \\
\text { (2001), UK }\end{array}$ & 5 yrs. & $\mathrm{C}$ & 20 & $<32$ & $<1200$ & 20 & NA & NA & Memory; Nonverbal ability \\
\hline $\begin{array}{l}\text { Hugues et al. } \\
\text { (2002), US }\end{array}$ & $3,6,12$ mos. & $C \& L$ & & 30.3 & NA & NA & NA & NA & Attention \\
\hline \multirow{3}{*}{$\begin{array}{l}\text { Rose et al. } \\
\text { (2003), US }\end{array}$} & \multirow{3}{*}{$5,7,12$ mos. } & \multirow{3}{*}{$\mathrm{L}$} & 59 & \multirow{3}{*}{29.6} & \multirow{3}{*}{1107} & 153 & \multirow{3}{*}{$38-42$} & \multirow{3}{*}{2500} & \multirow{3}{*}{$\begin{array}{l}\text { Visual recognition memory; } \\
\text { Processing speed; Short-term } \\
\text { memory capacity; Attention }\end{array}$} \\
\hline & & & 56 & & & 144 & & & \\
\hline & & & 50 & & & 126 & & & \\
\hline $\begin{array}{l}\text { Vicari et al. } \\
(2004), \text { I }\end{array}$ & $3-4$ yrs. & $\mathrm{C}$ & 19 & $29-34$ & $910-2300$ & 19 & $38-42$ & $>2800$ & $\begin{array}{l}\text { Attention; Spatial Working } \\
\text { Memory, Perception; IQ }\end{array}$ \\
\hline $\begin{array}{l}\text { Espy et al. } \\
\text { (2007), US }\end{array}$ & 3 yrs. & FS & 22 & $\begin{array}{l}<35 \& \\
>28\end{array}$ & $\begin{array}{l}>1250- \\
<2000\end{array}$ & NA & NA & NA & $\begin{array}{l}\text { Attention; Executive control; } \\
\text { Math skills }\end{array}$ \\
\hline $\begin{array}{l}\text { Hunnius et al. } \\
\text { (2008), NL }\end{array}$ & $\begin{array}{l}6,10,14,18 \\
22,26 \text { wks. }\end{array}$ & $\mathrm{L}$ & 10 & 29.6 & 1267 & 20 & NA & 3220 & $\begin{array}{l}\text { Attention development; } \\
\text { Sensorimotor processing }\end{array}$ \\
\hline \multirow{2}{*}{$\begin{array}{l}\text { Clark et al. } \\
\text { (2008), NZ }\end{array}$} & \multirow{2}{*}{2,4 yrs. } & \multirow{2}{*}{$\mathrm{C}$} & 39 & $<28$ & 1069 & \multirow{2}{*}{103} & NA & NA & Atte \\
\hline & & & 56 & $<34$ & & & NA & $N A$ & Attention \\
\hline $\begin{array}{l}\text { Sondaar et al. } \\
\text { (2008), NL }\end{array}$ & 5 yrs. & FS & 368 & $<32$ & $<1500$ & NA & NA & NA & $\begin{array}{l}\text { Cognitive development; } \\
\text { Language Development; } \\
\text { Academic performance; IQ }\end{array}$ \\
\hline $\begin{array}{l}\text { Feldman } \\
\text { (2009), IL }\end{array}$ & $\begin{array}{l}3,6,12, \\
24 \text { mos., } 5 \text { yrs. }\end{array}$ & $C \& L$ & 125 & $25-35$ & $<1750$ & NA & NA & NA & Executive function; Attention \\
\hline $\begin{array}{l}\text { Cornelieke et } \\
\text { al. (2009), NL }\end{array}$ & 6 yrs. & $\mathrm{C}$ & 50 & 28 & NA & 50 & NA & NA & $\begin{array}{l}\text { Inhibition: Work Memory, } \\
\text { Concepts generation }\end{array}$ \\
\hline Baron et al. & 3 yrs. & C & 20 & $<33$ & $<1000$ & 40 & $>37$ & $>2500$ & Spatial location Memory \\
\hline (2010), US & oyrs. & & 75 & $34-36$ & 2500 & - & - & - & Spatral Iocation Miemory \\
\hline $\begin{array}{l}\text { Dall'oglio et } \\
\text { al. (2010), I }\end{array}$ & 4 yrs. & $\mathrm{C}$ & 35 & $<33$ & 1250 & 50 & NA & 3459 & $\begin{array}{l}\text { Short-term Memory; Visual-motor } \\
\text { and constructive spatial abilities; } \\
\text { Visual processing }\end{array}$ \\
\hline & & & & $<28$ & $<1000$ & 74 & NA & $>2000$ & \\
\hline $\begin{array}{l}\text { Nordhov et al. } \\
\text { (2010), N }\end{array}$ & $3-5$ yrs. & $\mathrm{L}$ & 72 & $>28$ & $1000-1500$ & - & - & - & IQ; Cognitive development \\
\hline & & & & $>33$ & $>1500-2000$ & - & - & - & \\
\hline Evrard et al. & $1218 \mathrm{mos}$ & $C \& \mathrm{I}$ & 38 & $<30$ & NA & 38 & NA & NA & Coonitive reoulation \\
\hline (2011), F & 12,18 mos. & $C \propto L$ & 36 & $30-32$ & - & 38 & NA & $N A$ & Cognitive regulation \\
\hline $\begin{array}{l}\text { Woodward et } \\
\text { al. (2011), NZ }\end{array}$ & 4 yrs. & $\mathrm{C}$ & 107 & $<32$ & NA & 113 & NA & NA & Executive function \\
\hline & & & & & $<1000$ & & & & \\
\hline $\begin{array}{l}\text { Pitchford et al. } \\
\text { (2011), UK }\end{array}$ & $2-5$ yrs. & $\mathrm{C}$ & 54 & $<30$ & $1000-1500$ & 37 & NA & NA & $\begin{array}{l}\text { Cognitive development; } \\
\text { Attention. Memory: Process speed }\end{array}$ \\
\hline & & & & & $>1500$ & & & & \\
\hline $\begin{array}{l}\text { Roberts et al. } \\
\text { (2011), AU }\end{array}$ & 2,5 yrs. & $\mathrm{C}$ & 227 & $<30$ & $<1250$ & 78 & NA & NA & Cognitive skills \\
\hline $\begin{array}{l}\text { Perricone et al. } \\
\text { (2011), I }\end{array}$ & 5 yrs., 2 mos. & $\mathrm{C}$ & 50 & $<32$ & $1500-2500$ & 55 & NA & NA & Attention \\
\hline
\end{tabular}

*Abbreviation of country; $†$ Mean or range; L=Longitudinal, $\mathrm{C}=$ Comparative; FS=Follow up study; GA=Gestational age (mean or range) in weeks; $\mathrm{BG}=$ Birth weight (mean or range) in grams; $\mathrm{N}=$ Indicates data were not reported or not extractable. 


\section{Table 2 Communicative and linguistic development}

\begin{tabular}{|c|c|c|c|c|c|c|c|c|c|}
\hline \multirow{3}{*}{$\begin{array}{l}\text { Source and } \\
\text { country* }\end{array}$} & \multirow{3}{*}{$\begin{array}{l}\text { Age range } \\
\text { or mean }\end{array}$} & \multirow{3}{*}{$\begin{array}{l}\text { Study } \\
\text { design }\end{array}$} & \multicolumn{6}{|c|}{ Characteristic } & \multirow{3}{*}{ Communicative-linguistic variables } \\
\hline & & & \multicolumn{3}{|c|}{ Study group } & \multicolumn{3}{|c|}{ Control group } & \\
\hline & & & $\mathrm{n}$ & GA & BW & $\mathrm{n}$ & GA & BW & \\
\hline $\begin{array}{l}\text { Briscoe et al. } \\
\text { (1998), UK }\end{array}$ & $3-4$ yrs. & $\mathrm{C}$ & 26 & $<32$ & 1209 & 26 & $>37$ & NA & $\begin{array}{l}\text { Receptive vocabulary; Productive vocabulary; } \\
\text { Expressive language (information and sentence } \\
\text { length); Phonological short-term memory (digit } \\
\text { span and nonword repetition) }\end{array}$ \\
\hline $\begin{array}{l}\text { Luoma et al. } \\
\text { (1998), FI }\end{array}$ & 5 yrs. & $\mathrm{C}$ & 55 & $<32$ & 1394 & 55 & 39.7 & 3474 & $\begin{array}{l}\text { Language comprehension (discriminating phonemes, } \\
\text { verbal-logical concepts and relations between words); } \\
\text { Language production (verbal fluency, rapid naming, } \\
\text { words naming); Speech (repeat phonological } \\
\text { sequences, repeat words and nonwords) }\end{array}$ \\
\hline $\begin{array}{l}\text { Wolke \& Meyer } \\
\text { (1999), DE }\end{array}$ & 6 yrs. & $\mathrm{C}$ & 264 & $<32$ & 1288 & 264 & 39 & 3407 & $\begin{array}{l}\text { Grammatical rules (plural-singular rules); Language } \\
\text { comprehension (semantically inconsistent } \\
\text { sentences), Language production (sentence } \\
\text { production); Grammatical rules (understanding } \\
\text { grammatical structures); Articulation; Prereading } \\
\text { skills (rhyming, sound-to-word-matching, naming } \\
\text { of letters and numbers) }\end{array}$ \\
\hline $\begin{array}{l}\text { Briscoe et al. } \\
\text { (2001), UK }\end{array}$ & 5 yrs. & $\mathrm{C}$ & 20 & $<32$ & $<1200$ & 20 & NA & NA & Verbal ability \\
\hline $\begin{array}{l}\text { Therion et al. } \\
\text { (2004), USA }\end{array}$ & 1 to 3 days & $\mathrm{L}$ & 35 & $24-32$ & $<1500$ & 40 & $\begin{array}{l}39- \\
41\end{array}$ & $>3500$ & $\begin{array}{l}\text { Auditory recognition memory; Speech sound } \\
\text { discrimination }\end{array}$ \\
\hline $\begin{array}{l}\text { Jansson- } \\
\text { Verkasalo et al. } \\
(2004), \text { FI } \\
\end{array}$ & $\begin{array}{l}2 \& \\
4 \text { yrs. }\end{array}$ & FS & 17 & 28 & 1049 & 17 & 39.7 & 3617 & $\begin{array}{l}\text { Language comprehension; Naming; Auditory } \\
\text { discrimination }\end{array}$ \\
\hline $\begin{array}{l}\text { Jansson- } \\
\text { Verkassalo et al. } \\
(2004), \text { FI } \\
\end{array}$ & 5 yrs. & $\mathrm{C}$ & 12 & 29 & 1115 & 12 & 39 & 3770 & Object naming; Auditory processing \\
\hline $\begin{array}{l}\text { Foster-Cohen et } \\
\text { al. (2007), NZ } \\
\end{array}$ & 2 yrs. & $\mathrm{C}$ & 102 & $\begin{array}{l}<28 \\
28-32\end{array}$ & $<1500$ & 90 & $\begin{array}{l}38- \\
41\end{array}$ & 3591 & $\begin{array}{l}\text { Vocabulary size; Quality of word use; Morphological } \\
\text { and syntactic complexity }\end{array}$ \\
\hline $\begin{array}{l}\text { Marston et al. } \\
\text { (2007), UK }\end{array}$ & 2 yrs. & FS & 288 & $23-28$ & $<1500$ & NA & NA & NA & Vocabulary (number of words) \\
\hline $\begin{array}{l}\text { Slolt et al. } \\
\text { (2007), FI }\end{array}$ & 2 yrs. & $\mathrm{C}$ & 66 & 27 & $<1500$ & 87 & $>37$ & $>3500$ & $\begin{array}{l}\text { Vocabulary size; Vocabulary composition (social } \\
\text { terms, common nouns, predicates, grammatical } \\
\text { function words) }\end{array}$ \\
\hline $\begin{array}{l}\text { Espy et al. } \\
(2007), \text { US }\end{array}$ & 3 yrs. & FS & 22 & $\begin{array}{l}<35 \\
>28\end{array}$ & $\begin{array}{l}>1250 \\
<2000\end{array}$ & NA & NA & NA & Verbal skills \\
\hline $\begin{array}{l}\text { Sondaar et al. } \\
\text { (2008), NL }\end{array}$ & 5 yrs. & FS & 368 & $<32$ & $<1500$ & NA & NA & NA & Language Development \\
\hline $\begin{array}{l}\text { Wolke et al. } \\
\text { (2008), UK }\end{array}$ & 6 yrs. & $\mathrm{C}$ & 200 & $<25$ & NA & 160 & NA & NA & $\begin{array}{l}\text { Receptive and expressive language; Auditory } \\
\text { comprehension; Expressive communication; } \\
\text { Articulation of sounds }\end{array}$ \\
\hline $\begin{array}{l}\text { Van Lierde et al. } \\
(2009), \mathrm{BE}\end{array}$ & 3 yrs. & $\mathrm{C}$ & 15 & $<27$ & $<1500$ & 19 & 39 & 3500 & $\begin{array}{l}\text { Receptive language; Expressive language (vocabulary, } \\
\text { semantics, morphosyntaxis) }\end{array}$ \\
\hline $\begin{array}{l}\text { Cornelieke et al. } \\
(2009), \text { NL }\end{array}$ & 6 yrs. & $\mathrm{C}$ & 50 & 28 & NA & 50 & NA & NA & Verbal fluency; Concepts generation \\
\hline $\begin{array}{l}\text { Cattani et al. } \\
(2010), \text { I }\end{array}$ & $\begin{array}{l}12 \& 24 \\
\text { mos. }\end{array}$ & $\mathrm{C}$ & 12 & 31.8 & 1845 & 59 & NA & NA & $\begin{array}{l}\text { Action/gesture production; Word comprehension, } \\
\text { Word production }\end{array}$ \\
\hline $\begin{array}{l}\text { Dall'oglio et al. } \\
\text { (2010), I }\end{array}$ & 4 yrs. & $\mathrm{C}$ & 35 & $<33$ & 1250 & 50 & NA & 3459 & Language \\
\hline
\end{tabular}


Continuation of Table 2 Communicative and linguistic development

\begin{tabular}{|c|c|c|c|c|c|c|c|c|c|}
\hline \multirow{3}{*}{$\begin{array}{l}\text { Source and } \\
\text { country* }^{*}\end{array}$} & \multirow{3}{*}{$\begin{array}{l}\text { Age range } \\
\text { or mean }\end{array}$} & \multirow{3}{*}{$\begin{array}{l}\text { Study } \\
\text { design }\end{array}$} & \multicolumn{6}{|c|}{ Characteristic } & \multirow{3}{*}{ Communicative-linguistic variables } \\
\hline & & & \multicolumn{3}{|c|}{ Study group } & \multicolumn{3}{|c|}{ Control group } & \\
\hline & & & $\mathrm{n}$ & GA & BW & $\mathrm{n}$ & GA & BW & \\
\hline $\begin{array}{l}\text { Kern \& Gayraud } \\
\text { (2011), F }\end{array}$ & $\begin{array}{l}24-26 \\
\text { mos. }\end{array}$ & $\mathrm{C}$ & 323 & $\begin{array}{l}<28 \\
28-32 \\
33-36\end{array}$ & NA & 166 & $>36$ & NA & $\begin{array}{l}\text { Vocabulary size; Grammatical categories (proportion } \\
\text { of nouns, of predicates, of closed class, of others); } \\
\text { Morphosyntactic development (MaxLu) }\end{array}$ \\
\hline $\begin{array}{l}\text { Pitchford et al. } \\
\text { (2011), UK }\end{array}$ & $2-5$ yrs. & $\mathrm{C}$ & 54 & $<30$ & $\begin{array}{l}<1000 \\
1000- \\
1500 \\
>1500\end{array}$ & 37 & NA & NA & Cognitive development; Language \\
\hline $\begin{array}{l}\text { Howard et al. } \\
\text { (2011), AU }\end{array}$ & $2 \& 5$ yrs. & $\mathrm{L}$ & 227 & $<30$ & $<1250$ & NA & NA & NA & $\begin{array}{l}\text { Expressive language skills; Receptive language } \\
\text { skills; Knowledge of number concepts and symbols; } \\
\text { Knowledge of letters and words }\end{array}$ \\
\hline $\begin{array}{l}\text { Roberts et al. } \\
\text { (2011), AU }\end{array}$ & 2,5 yrs. & $\mathrm{C}$ & 227 & $<30$ & $<1250$ & 78 & NA & NA & Expressive and receptive language \\
\hline
\end{tabular}

*Abbreviation of country; L=Longitudinal, C=Comparative; FS=Followup study; GA=Gestational age (mean or range) in weeks; BW=Birth weight (mean or range) in grams; $\mathrm{NA}=$ Indicates data were not reported or not extractable.

Table 3 Personal and social development

\begin{tabular}{|c|c|c|c|c|c|c|c|c|c|}
\hline \multirow{3}{*}{$\begin{array}{l}\text { Source and } \\
\text { country* }\end{array}$} & \multirow{3}{*}{$\begin{array}{l}\text { Age range } \\
\text { or mean }\end{array}$} & \multirow{3}{*}{$\begin{array}{l}\text { Study } \\
\text { design }\end{array}$} & \multicolumn{6}{|c|}{ Characteristic } & \multirow{3}{*}{ Personal and social variables } \\
\hline & & & \multicolumn{3}{|c|}{ Study group } & \multicolumn{3}{|c|}{ Control group } & \\
\hline & & & $\mathrm{n}$ & GA & BW & $\mathrm{n}$ & GA & $\mathrm{n}$ & \\
\hline $\begin{array}{l}\text { Beckwith } \\
\text { \& Rodning } \\
\text { (1996), US }\end{array}$ & $2-5$ yrs. & FS & 51 & 30,9 & 1448 & NA & NA & NA & $\begin{array}{l}\text { Child development social problem solving; } \\
\text { Mother-child interactions }\end{array}$ \\
\hline $\begin{array}{l}\text { Landry et al. } \\
\text { (1998), US }\end{array}$ & $\begin{array}{l}6,12, \\
24 \text { mos., } \\
3 \text { yrs., } 6 \text { mos., } \\
4 \text { yrs., } 6 \text { mos. }\end{array}$ & $\begin{array}{l}\mathrm{C} \& \\
\mathrm{~L}\end{array}$ & $\begin{array}{l}73 \\
114\end{array}$ & $<36$ & $<1600$ & 114 & $\begin{array}{l}37- \\
42\end{array}$ & NA & $\begin{array}{l}\text { Child Social competence: Responsiveness and } \\
\text { initiations maternal behaviors: maintaining and } \\
\text { directness, and warm sensitivity }\end{array}$ \\
\hline $\begin{array}{l}\text { Landry et al. } \\
\text { (2000), US }\end{array}$ & $\begin{array}{l}6,12, \\
24 \text { mos., } \\
3 \text { yrs., } 6 \text { mos., } \\
4 \text { yrs., } 6 \text { mos. }\end{array}$ & $\begin{array}{l}\mathrm{C} \& \\
\mathrm{~L}\end{array}$ & 73 & $<36$ & $<1600$ & 114 & $\begin{array}{l}37- \\
42\end{array}$ & NA & $\begin{array}{l}\text { Child social competence: Responsiveness and } \\
\text { initiations; Maternal behaviors: Maintaining and } \\
\text { directness and warm sensitivity }\end{array}$ \\
\hline $\begin{array}{l}\text { Assel et al. } \\
\text { (2002), US }\end{array}$ & $3 \& 4$ yrs. & $\mathrm{C}$ & 180 & 29,7 & 1117 & 112 & NA & NA & $\begin{array}{l}\text { Social interactions; Behavior and emotional } \\
\text { problems; Parenting behavior: Emotional } \\
\text { functioning (parental stress): Maternal child- } \\
\text { rearing history }\end{array}$ \\
\hline $\begin{array}{l}\text { Hugues et al. } \\
\text { (2002), US }\end{array}$ & $\begin{array}{l}3,6 \& \\
12 \text { mos. }\end{array}$ & $\begin{array}{l}\mathrm{C} \& \\
\mathrm{~L}\end{array}$ & & 30,3 & NA & NA & NA & NA & Temperament \\
\hline $\begin{array}{l}\text { Delobel-Ayoub } \\
\text { et al. (2006), F }\end{array}$ & 3 yrs. & FS & 1228 & $22-33$ & NA & 447 & NA & NA & Behavioral Problems and difficulties \\
\hline $\begin{array}{l}\text { Lavta } \\
(2008), \text { FI }\end{array}$ & $5-6$ yrs. & $\mathrm{C}$ & 28 & NA & $<2500$ & 39 & NA & NA & $\begin{array}{l}\text { Maternal recollections of the birth experiences; } \\
\text { Behavioral and emotional problems }\end{array}$ \\
\hline $\begin{array}{l}\text { Cevasco } \\
\text { (2008), US }\end{array}$ & Newborn & PPT & 20 & 32 & $<2500$ & 54 & NA & NA & $\begin{array}{l}\text { Adjustment maternal (to infant and lifestyle } \\
\text { changes); Maternal Bonding; Maternal value of } \\
\text { music for their babies; Use of CDs; Post-birth } \\
\text { complications; Comments about the survey }\end{array}$ \\
\hline $\begin{array}{l}\text { Clark et al. } \\
\text { (2008), NZ }\end{array}$ & $2 \& 4$ yrs. & C & $\begin{array}{l}39 \\
56\end{array}$ & $\begin{array}{l}<28 \\
<34\end{array}$ & 1,069 & 103 & NA & NA & $\begin{array}{l}\text { Emotional and behavioral regulation: Infant's and } \\
\text { parent's behavior in parent-child interactions; } \\
\text { Infant Development; Parent's self-regulation; } \\
\text { Infant clinical and neuroanatomical and family } \\
\text { social background; Parenting (sensitivity and } \\
\text { intrusiveness); Early intervention }\end{array}$ \\
\hline
\end{tabular}




\begin{tabular}{|c|c|c|c|c|c|c|c|c|c|}
\hline \multirow{3}{*}{$\begin{array}{l}\text { Source and } \\
\text { country* }\end{array}$} & \multirow{3}{*}{$\begin{array}{l}\text { Age range } \\
\text { or mean }\end{array}$} & \multirow{3}{*}{$\begin{array}{l}\text { Study } \\
\text { design }\end{array}$} & \multicolumn{6}{|c|}{ Characteristic } & \multirow{3}{*}{ Personal and social variables } \\
\hline & & & \multicolumn{3}{|c|}{ Study group } & \multicolumn{3}{|c|}{ Control group } & \\
\hline & & & $\mathrm{n}$ & GA & $\mathrm{BW}$ & $\mathrm{n}$ & GA & $\mathrm{n}$ & \\
\hline $\begin{array}{l}\text { Feldman } \\
\text { (2009), IL }\end{array}$ & $\begin{array}{l}3,6,12 \\
24 \text { mos. } \& \\
5 \text { yrs. }\end{array}$ & $\begin{array}{l}\mathrm{C} \& \\
\mathrm{~L}\end{array}$ & 125 & $25-35$ & $<1750$ & NA & NA & NA & $\begin{array}{l}\text { Cardiac vagal tone and sleep-wake cyclicity; } \\
\text { Infant medical risk; Emotion regulation; } \\
\text { Self-regulation: behavior adaptation or problems, } \\
\text { executive functions and self-restraint }\end{array}$ \\
\hline $\begin{array}{l}\text { Korja } \\
\text { (2009), FI }\end{array}$ & $6 \& 12$ mos. & $\mathrm{C}$ & 70 & $<32$ & $<1501$ & 46 & NA & NA & $\begin{array}{l}\text { Mother-infant interaction; Maternal } \\
\text { representations; Maternal depression }\end{array}$ \\
\hline $\begin{array}{l}\text { Spittle et al. } \\
\text { (2009), AU }\end{array}$ & 2 yrs. & $\mathrm{C}$ & 188 & $<30$ & $<1250$ & 70 & NA & NA & $\begin{array}{l}\text { Externalizing, internalizing and dysregulation } \\
\text { problems; Social-emotional competencies } \\
\text { Sex, birth weight, gray/white matter } \\
\text { abnormalities postnatal corticosteroids }\end{array}$ \\
\hline $\begin{array}{l}\text { Treyvaud et al. } \\
\text { (2009), AU }\end{array}$ & 2 yrs. & $\mathrm{C}$ & 177 & $<30$ & $<1250$ & 69 & NA & NA & $\begin{array}{l}\text { Social risk; Parenting behaviors; Parent-child } \\
\text { synchrony; Social-emotional development }\end{array}$ \\
\hline $\begin{array}{l}\text { Treyvaud et al. } \\
\text { (2010), AU }\end{array}$ & 2 yrs. & $\mathrm{C}$ & 177 & $<30$ & $<1250$ & 69 & NA & NA & $\begin{array}{l}\text { Parental Mental health; Social-emotional } \\
\text { development; Positive and Negative affect; Social } \\
\text { risk }\end{array}$ \\
\hline $\begin{array}{l}\text { Spittle et al. } \\
\text { (2010), AU }\end{array}$ & 2 yrs. & PPT & 61 & $<30$ & NA & 59 & $<30$ & NA & $\begin{array}{l}\text { Behavior and emotional regulation; Symptoms of } \\
\text { anxiety or depression }\end{array}$ \\
\hline $\begin{array}{l}\text { Roberts et al. } \\
(2011), \mathrm{AU}\end{array}$ & $2 \& 5$ yrs. & $\mathrm{C}$ & 227 & $<30$ & $<1250$ & 78 & NA & NA & $\begin{array}{l}\text { Emotional well-being and social competence } \\
\text { (strengths and difficulties) }\end{array}$ \\
\hline $\begin{array}{l}\text { Evrard et al. } \\
\text { (2011), F }\end{array}$ & $12 \& 18$ mos. & $\begin{array}{l}\mathrm{C} \& \\
\mathrm{~L}\end{array}$ & $\begin{array}{l}74 \\
38 \\
36 \\
\end{array}$ & $\begin{array}{l}<30 \\
30-32\end{array}$ & NA & 38 & NA & NA & $\begin{array}{l}\text { Emotional regulation; Social behavior and social } \\
\text { involvement }\end{array}$ \\
\hline $\begin{array}{l}\text { Perricone et al. } \\
\text { (2011), I }\end{array}$ & $\begin{array}{l}5 \text { years } \& \\
2 \text { mos. }\end{array}$ & $\mathrm{C}$ & 50 & $<32$ & $\begin{array}{l}1500- \\
2500\end{array}$ & 55 & NA & NA & Temperament \\
\hline $\begin{array}{l}\text { Gray et al. } \\
\text { (2012), AU }\end{array}$ & 4 mos. & $\mathrm{C}$ & 124 & $<30$ & 1116 & 120 & $>37$ & NA & $\begin{array}{l}\text { Parenting Stress; Postnatal Depression; Dyadic } \\
\text { Adjustment; Temperament }\end{array}$ \\
\hline $\begin{array}{l}\text { Ravn et al. } \\
\text { (2012), N }\end{array}$ & $\begin{array}{l}1,4,6 \& \\
12 \text { mos. }\end{array}$ & $\begin{array}{l}\mathrm{C} \& \\
\mathrm{~L}\end{array}$ & 56 & 33.3 & 1937 & 50 & 33.0 & 1894 & $\begin{array}{l}\text { Depression; Parenting Stress; Infant Behavior } \\
\text { (Temperament); Infant's communication skills } \\
\text { (social interactions) }\end{array}$ \\
\hline $\begin{array}{l}\text { Tooten et al. } \\
\text { (2012), NL }\end{array}$ & $6 \& 24$ mos. & $\begin{array}{l}\text { PPT } \\
\& \mathrm{~L}\end{array}$ & 70 & $<32$ & $\begin{array}{l}\text { ELBW } \\
\text { VLBW }\end{array}$ & $\begin{array}{l}140 \\
70 \\
70\end{array}$ & $\begin{array}{l}>37 \\
32- \\
37\end{array}$ & NA & $\begin{array}{l}\text { Parent-child interactions; Parental bonding; } \\
\text { Temperament infant; Behavior infant; } \\
\text { Social-Emotional Development }\end{array}$ \\
\hline
\end{tabular}

*Abbreviation of country; L=Longitudinal, $\mathrm{C}=$ Comparative; FS=Follow up study; PPT=Pre-Post test; GA=Gestational age (mean or range) in weeks; $\mathrm{BW}=\mathrm{Birth}$ weight (mean or range) in grams; $\mathrm{NA}=$ Indicates data were not reported or not extractable; $\mathrm{ELBW}=$ extremely low birth weight; VLBW=very low birth weight.

hension than full-term children, when taking into account maternal education and cognitive level (45). However, no differences were found between the two groups in lexical production (45).

Jansson-Verkasalo et al. (62) found that after 5 years preterm children obtained poorer scores in object naming and syllable discrimination tasks than full-term children. Luoma et al. (63) also found that at this age, preterm infants had poorer results than full- term children in naming words and understanding concepts tasks, but found no differences in global measures of spoken language comprehension and expression. Similarly, Roberts et al. (48) found that at this age, preterm children had problems in receptive and expressive language, independent of social risk. They also present problems in vocabulary and spoken language grammar skills (47), and obtain lower scores in listening comprehension and expressive communica- 
tion (40). The problems with vocabulary and morphosyntactic skills that preterm children manifest at this age are considered predictors of cognitive problems (50).

It has also been found that 6-year-old preterm children obtain lower scores than fullterm children in linguistic skills (semantics, grammar, and phonology), in pre-reading skills, such as rhyme, sound-word pairing and naming numbers and letters (64), and in verbal fluency when controlling for IQ (52). Wolke et al. (65) found that preterm children at this age are more likely to have receptive and expressive language problems, speech problems and overall school difficulties than full-term children.

\section{Personal and social development studies}

The following results have been reported in studies on personal and emotional development from birth to 12 months of age. Cevasco (66) found that after birth, children, who had heard recordings of their mothers when they were not present in the hospital, were discharged on average 2 days earlier than the control group, although the differences were not statistically significant. They stopped crying and were calmed by listening to their mother singing, but did not display smiling behavior, which full-term infants displayed. Tooten et al. (67) analyzed parent-infant interactive behavior in term, very and extremely preterm infants in order to evaluate the effectiveness of video interaction guidance in parents, to prevent adverse parent-infant interaction. Hughes et al. (35) observed changes in some temperament characteristics between 6 weeks and 12 months of life: at 6 weeks the infants were significantly less rhythmic, at 6 months they were less adaptable, and at 12 months they were less persistent. The changes in approaching and intensity suggest that preterm children become more energetic during the first 6 months. Compared to the standardized population, they only differed in persistence at 12 months; that is, they spent less time in activities, were less likely to persist in an activity when encountering an obstacle, were more passive, and less involved with their environment (35). Feldman (36) found that high-risk preterm children had poorer physiological regulation and a poorer sleepwake cycle, more negative emotions at 3, 6 and 12 months, and lower delayed response at 12 months, but that there were no differences between the groups at 12 months, or in adaptive behavior or self-control between 12 months and 2 years. Evrard et al. (37) found that preterm children in this period presented different emotional regulation profiles then full-term infants. The very premature children also displayed a lower frequency in social behaviors directed toward others, with less social involvement. Ravn et al. (68) used a parentreport instrument to evaluate infant temperament (i.e. activity level, distress to limitations, approach, smile and laughter and soothability) and infant communication skills (i.e. behavior regulation/request). At 6 and 12 months, mothers reported significantly less smiles and laughter in their children. At 12 months they reported less infant activity. No significant differences were found at 6 and 12 months in distress regarding limitations, approach, and duration of orientation or sooth ability. Also, Gray et al. (69) analyzed child temperament during the first year of life comparing preterm and full-term children. The temperament of the preterm infants was similar to the term infants (69).

Clark et al. (44) found that preterm children aged between 2 and 5 years displayed poorer self-regulation. They reported that at 4 years these children were more likely to present difficulties in emotional regulation and suggested that these difficulties become more visible to parents as the children mature and parental expectations increase (44). On the other hand, they found no differences 
between groups in terms of positive or negative affect during parent-child conflict resolution. Although no differences were found in the children's ability to move from one task to another, there were differences in levels of persistence that were demonstrated during problem solving tasks. Spittle et al. (70) found that preterm children at 2 years presented more problems in internalizing behavior (withdrawal, sadness, enjoyment, fear), in behavior management (negative emotions, difficulties with eating and sleeping) and in social competence (acceptance of norms, cooperation, social games, and imitating other children). However, no differences were found compared to full-term children in externalizing behaviors (motor activity, defiance, and aggression). Although in a subsequent work by Spittle et al. (5) no differences were found in other developmental domains, differences were found in the emotional-social domain (5). Thus, children in the new intervention group were described by their caregivers as exhibiting less externalizing and dysregulation behaviors and increased competence than those in the control group. No differences between groups were found in the internalizing domain. The caregivers of the children in the new intervention group reported less anxiety and depression (5).

Treyvaud et al. (71) and Roberts et al. (48) published key studies. Treyvaud et al. (71) found that $18 \%$ of preterm children were within the high-risk range in social-emotional competence, $9 \%$ in externalizing behavior, and $11 \%$ in internalizing behavior. Boys obtained significantly lower scores than girls, but subsequently no differences were found between genders in mother-child synchrony. In a later publication (72), the same authors found that preterm children presented socialemotional difficulties and an increased risk of dysregulation in relation to positive and negative affect. Roberts et al. (48) found that preterm children were more likely to have vulnerabilities in multiple domains of school readiness, independent of social risk. They obtained lower scores than their full-term peers in social-emotional skills, social competence and emotional wellbeing. Their results show that the preterm children had standard scores between 0.5 and 1.0 SD below those of the control group, in all domains of school readiness. Compared to $16 \%$ of the control group, $44 \%$ of the preterm children showed increased vulnerability in more than one domain of school readiness (48).

The EPIPAGE study (19) investigated behavioral problems in preterm children. They found that at 3 years of age these children are more likely to have behavioral difficulties (problems with peers, behavioral problems, and hyperactivity) than full-term children, even after controlling for the influence of demographic characteristics and neonatal complications. Beckwith and Rodning (73) analyzed mother-child interaction and children's social competence from 2 to 5 years of age (maternal responsiveness, infant irritability and social skills) and the results suggest that children born at higher birth weights and with longer gestation were more likely to be more irritable than those born at very low birth weights. Also, infant negative hedonic tone (infant affect) at 13 months showed significant correlations with outcomes at 2, 3 and 5 years. Assel et al. (74) compared preterm and full-term children at 3 and 4 years old in social initiations and social problems, and they analyzed the effects of parental variables such as: parenting, emotional functioning and maternal childrearing history. At 4 years preterm children have less social skills and more social problems.

Feldman (36) conducted a longitudinal study on preterm children and found that they had poorer performance at 5 years in inhibition and multimodal coordination. However, no differences were found between groups in adaptive behavior or self-control. 
Similarly, Landry et al. (39) found that preterm children were less initiating in social interactions than their full-term peers between 2 and 4.5 years of age. Landry et al. (75) also found that at 4.5 years, preterm children differed in their levels of play and social initiating skills from full-term children, who engaged in more pretend play and needed fewer trials to succeed in tasks. No differences were found between preterm and full-term children in their patterns of goal-directed behavior and social initiating skills.

Regarding children at 5-6 years of age, Lavta (76) found that preterm children displayed clinical behavioral and emotional symptoms. On the other hand, Feldman (36) found no differences in adaptive behavior, although this author reported more difficulties in inhibition and multimodal coordination. Perricone and Morales (49) found that at these ages preterm children present fewer emotional problems than cognitive ones, although they present problems in other social abilities. During this period, preterm children are temperamentally strongly disposed to express positive feelings, and have a strong tendency to seek others and be sociable, compared to full-term children; that is, they are more likely to express positive feelings and experiences. Full-term children had low scores on emotional reactivity scales and were thus defined as having a calm temperament profile. The authors found no evidence among preterm children at 5 years of age of a so-called "temperament syndrome", that is, children who are more emotive, sociable and patient than full-term children, but less directed and reactive to frustration.

\section{Discussion}

The aim of this review was to describe psychological development in three areas (the cognitive, communicative-linguistic and social-emotional domains) of very preterm children from 0 to 6 years of age, to learn if there are problems and if these difficulties remain, decrease or increase over time up to 6 years of age.

In relation to cognitive development, the research found global cognitive delays in children born prematurely, but the specific cognitive processes most affected are memory from 2 to 6 years $(40,42,43,45,47,48$, $52)$, attention from the first year of birth (35, $36,38,41,44)$ and visual-spatial processing at 3-4 years $(42,43,45)$. However, there is no consensus on the degree of impairment of memory and executive function, because some studies find they are not affected in the first 2 years (37), and other studies find worse scores at 5 and 6 years $(48,52)$. The majority of these studies highlight that the white matter and gray matter play a crucial role in general cognitive development, and executive function in particular. Increased damage to white matter, as well as decreased volume, lead to decreased cognitive ability (46). Many authors emphasize the importance of early neuropsychological examinations of low birth-weight preterm children since, due to their condition, they are at increased risk of a wide variety of neuropsychological deficits, including of spatial location memory and attention, during the first years ( $0-6$ years). The importance of early assessment and intervention is highlighted.

Studies on communicative - linguistic development found that preterm children have problems in auditory discrimination in the first days of life, and at 4 and 5 years old (53, $55,62)$, problems in grammar skills at $2,3,5$ and 6 years old $(47,50,55,57,59,61,64)$, problems in naming objects and words at 4 and 5 years old $(55,62,63,64)$ and pre-reading skills at 6 years (64). Other studies found that these children have no problems in lexical production at 4 years old (62). However, there are no conclusive data about problems in expressive and receptive language develop- 
ment in general, vocabulary size and communication skills. Some studies found that preterm children have problems in expressive and receptive language development in general in the first two years of life, and from 2 to 6 years of age $(40,48,52,54,61,62,65)$. One study found that preterm children have no problems in expressive and receptive language development in general at 5 years (63) and other study found that these children have problems in some specific components of expressive language (sentences), but they do not have problems in other components (information) at 3 years old (60). In vocabulary, some studies found that preterm children have problems at 2, 3, 5 and 6 years old $(41,47,50,57,60,62,64)$ and other studies found that they have no problems $(58,59$, 60 ). Some studies found that preterm children have problems in communication skills (verbal) in the first two years of life $(17,54)$, but others found that these children have no problems in communication skills (nonverbal) at 3 years old (60). Some studies have suggested that there is an association between vocabulary problems and perinatal hypoxia, a condition experienced by many preterm children (41). Lower maternal education levels, poorer communication skills and white matter abnormalities have been suggested as good predictors of language development in preterm children over the long term (17), and have been closely associated with potential cognitive function deficits $(50,65)$. These results suggest that possible linguistic problems in preterm children are more evident at earlier ages, and the influence of maternal education and poorer maternal communication skills are strongly related to the fact that these difficulties increase with time. For these reasons, the importance of primary attention from health services to the families of premature children is evident, in order to promote skills in the mothers that affect the language development of their offspring.
In relation to social and personal development studies, the reviewed studies found that preterm children have problems in social adjustment in the first months $(35,68)$, in task persistence also at 4 years $(35,44)$, in emotional regulation and social interactions from the first year of life $(37,39,44,68,71)$, in emotional adjustment $(48,68)$, in the development of secure attachment and social competence in the early years $(5,48,71)$, in internalizing behavior at two years (such as shyness, fear, anxiety, depression) $(5,71)$ and behavioral difficulties, such as hyperactivity at 3, 5 and 6 years (76). However, there is no agreement between the studies regarding externalizing behavior problems, such as aggression, hyperactivity and defiance, or emotional expression and regulation difficulties. Some studies suggest that preterm children have these difficulties $(5,36,48,71)$, while other studies suggest that preterm children have no problems with temperament at 12 months (69), in adaptive behavior and in self-control at 2 and 5-6 years (36), in effective problem solving with parents (44), or in externalizing behavior at two years (5). They also do not seem to present temperamental syndrome at five years, when children are most emotional, social and patient, less directive and reactive to frustration (49). In these studies great variability was observed, depending on the definition of the study variables and the instruments used. However, there is a greater consensus that an early attachment relationship between mother and child was one of the most important variables in the presence or absence of the difficulties that these children had.

In other reviews conclusions were found similar to the majority of our studies. Along this line, Mulder et al. (4) confirmed using meta-analysis that executive function and attention were areas of weakness for preterm children. The review showed the extent of difficulties that were influenced by gestation- 
al age. Specifically, gestational age was related to more difficulties in selective and sustained attention and inhibition (in cognitive areas), and phonemic fluency problems and shift skills (in communicative-linguistic areas). Likewise, Saavalainen et al. (77) showed that spatial working memory performance is related to gestational age. A similar pattern has been shown previously for IQ and gestational age $(27,78,79)$. In a meta-analysis Van Noort-van der Spek et al. (22) found scores significantly lower in language function tests in preterm children compared with term children. Along the same lines, in a systematic review, Glennis and Richardson (28) found the effect of preterm birth/very low birth weight on health-related quality of life seemed to diminish over time. The impact of low birth weight and gestational age is greatest during the younger years, but the influence also extends into adolescence and adulthood. In other words, all these studies found the risk conferred by prematurity for deficits in these skills diminishes with increasing gestational age.

This review is limited by the heterogeneity of the populations included in the review. The gestational age in different studies was not homogeneous. To control this heterogeneity studies were excluded with samples that included preterm children with handicaps in their development. This could make the generalization of the results found difficult. Another limitation was that all the studies reviewed were in English. Perhaps if we had included studies in other languages, the results obtained would have been different or we would be able to generalize our conclusions more widely. In future research it would be interesting to consider studies in other languages in order to generalize the results more widely. Also could be interesting to include studies of all preterm children (with and without handicaps), to compare if development is worse in preterm children with handicaps. In this context, we would like to undertake a systematic review with preterm children at school age. Finally, we are planning to do experimental research to compare the development of different groups of preterm children (preterm children, very preterm children and extremely preterm children) with different birth-weights, to analyze the kind of differences that may be observed between them.

\section{Conclusion}

The main characteristics of this review of a total of 47 articles are the following: The majority of these studies have been published since 2005. The majority of the studies on cognitive development that we analyzed were conducted first in the USA, followed by the Netherlands, Italy and the UK. The most frequent study design was comparative, in the majority of studied areas of development, followed by longitudinal-comparative studies, longitudinal, follow-up studies, and pretest-posttest studies. The review study shows a variety of designs of comparative studies, longitudinal studies and follow up studies. All of this made the comparison of the studies difficult. For this reason, it is important to propose research to decrease the difficulties found in these areas and improve adaptation of children in the early years of school. The comparative studies typically used children born at term as the control group and only rarely were other preterm groups used. There were very few studies comparing different types of preterm children, and very few studies comparing preterm groups in relation to the social-emotional context. Instruments used in reviewed studies also were varied and difficult to compare. In the cognitive and communicative-linguistic domains the variables were assessed using standardized measuring instruments. In contrast socialemotional variables were usually assessed using observational measures, although the 
studies increasingly attempted to design and test more objective instruments. The gestational age in different studies was not homogeneous and was not in accord with de criteria of OMS and ICD-10. In the majority of studies, the gestational age of participants was $<30-32$ weeks, followed by $<28$ weeks in studies of communicative-linguistic development and $<36$ weeks in studies of socialemotional development. The majority of birth weights were between 1000-2499 gr., followed by $<1000$ gr., and then different categories of birth weight $(<1250,<1500,1500$, etc.). Not all of them fall into categories established by ICD-10 was. We did not found common criteria between the studies in relation to the selection of the samples, with respect to gestational age and weight of prematurity as established by the OMS or ICD- 10 .

The main differences and similarities found in the studies were the following: several of these studies found a close association between the different developmental domains; many studies investigated the influence of prematurity on cognitive development and its relationship to language development (40, 41, 45, 47, 48, 52, 58). Other studies compared the cognitive component and social-emotional aspects $(35,36$, $37,44,48,49,73,75)$ and yet others compared communicative-linguistic skills and emotional development $(48,74)$. However, no studies were found that investigated the influence of prematurity on all the developmental domains at the same time, in the same research. In the studies reviewed, the period of gestation and birth weight were important predictors of future health issues. The shorter the child's period of gestation and the lower the birth weight, the greater the risk of cognitive, communicative and social deficits $(5,42,45,46,47,48,50,52,56,58,66)$. Other studies drew attention to the importance and influence of perinatal risk factors (medical complications during birth, hypox- ia, hypertension, white matter abnormalities) in preterm children, particularly regarding their cognitive and language development $(17,41,46,69)$. Similarly, the influence of contextual risk factors (socioeconomic status, educational level and parental interactive style, mother-child attachment and type of attachment, parental and maternal emotional adjustment and mental health, negative maternal anxiety and displayed affect, support networks, early parenthood, expectations and characteristics of the proposed task) on the cognitive, communicative-linguistic, and especially emotional development, of preterm children has also been highlighted $(17,39$, $45,49,66,68,69,71,72,74,75)$. The majority of these studies investigated the influence of sociocultural factors as covariables on the psychological development of preterm children. There is also consensus that from 2 years old onwards the influence of biological risk variables decreases, whereas the influence of psychosocial variables increases in preterm children. In summary, some studies did not show a consensus in the determined aspects, but it appears that most agree that the most important predictors in the evolution of development of premature children were white matter, birth-weight and gestational age, on the biological plane; and maternal education, the maternal communication skills and early attachment, on the contextual plane.

There are few longitudinal studies that address the multidimensional aspects of all the developmental areas, rather than just specific aspects of development. Further studies should include different measuring instruments (observation, self-reports, standardized tests, medical tests, etc.), use larger samples, and take into account the influence of different contexts or situations (in-hospital period, educational styles, attachment models, type of parent-child interaction, emotional adjustment of parents, etc.) to compare the devel- 
opment of different types of preterm children $(67,72,80)$.

Finally, many studies highlight the importance of studying these children from a multidisciplinary perspective (e.g., psychology, pediatrics, neonatology and gynecology), with the objective of preventing physical and psychological problems. In this context, it is important to inform pediatricians, psychologists, and other associated professionals, of the large number of papers that have highlighted the importance of early care (early intervention) as a means to reduce or prevent the risk of cognitive, communicative and social-emotional problems in these children $(42,45,47,48,50,51)$. The majority of these studies highlight early care as a predictor of successful outcomes in the cognitive $(42,47,48,50,51,67)$ communicativelinguistic $(45,47)$ and emotional domains $(48,70,71)$. Early intervention is the most important way to prevent and/or reduce academic, communicative and social-emotional (anxiety/depression) problems, and improve long-term adaptation to the environment, self-regulation, communication skills, social competence and academic performance in general. In this sense, the scientific and professional community could also benefit from further studies regarding the influence of early care on preterm children in all areas of psychological development and family wellbeing.

\section{References}

1. World Health Organization [homepage on the internet]. Preterm birth. Fact sheet N³63, 2013. [cited 2014 August 17]. Available from: http:// www.who.int/mediacentre/factsheets/fs363/es/.

2. International Classification Disease (ICD-10) [homepage on the internet]. Disorders relating to short gestation and low birthweigth. 2013 [cited 2014 August 19]. Available from: http:// www.icd9data.com/2013/Volume1/760-779/ 764-779/765/default.htm.
3. Aarnoudse-Moens CS, Weisglas-Kuperus N, Van Goudoever JB, Oosterlaan J. Meta-analysis of Neurobehavioral outcomes in very preterm and/ or very low birth weight children. Pediatrics. 2012;124:717-28.

4. Mulder H, Pichford NJ, Hasgger MS, Marlow N. Development of executive function and attention in preterm children: A systematic review. Dev Neuropsychol. 2009;34:393-421.

5. Spittle AJ, Anderson PJ, Lee KJ, Ferretti C, Eeles A, Orton J, et al. Preventive care at home for very preterm infants improves infant and caregiver outcomes at 2 years. Pediatrics. 2010;126:170-78.

6. Taubman-Ben-Ari O, Findler L, Kuint J. Personal Growth in the wake of stress: the case of mothers of preterm twins. J Psychol. 2010;144(2):185-204.

7. Back SA, Riddle A, McClure MM. Maturationdependent vulnerability of perinatal white matter in premature birth. Stroke. 2007;38:724-30.

8. Horbar JD, Badger GJ, Carpenter JH, Fanaroff AA, Kilpatrick S, LaCorteM, et al. Trends in mortality and morbidity for very low birth weight infants, 1991-1999. Pediatrics. 2002;110:143-51.

9. Mercier CE, Dunn MS, Ferrelli KR, Howard DB, Soll RF. Neurodevelopmental outcome of extremely low birth weight infants from the Vermont Oxford Network: 1988-2003. Neonatology. 2010;97:329-38.

10. Costeloe K, Hennessy E, Gibson A, Marlow N, Wilkinson AR. The EPICure Study: outcomes to discharge from hospital for infants born at the threshold of viability. Pediatrics. 2000;106:659-71.

11. Allin M, Matsumoto H, Santhouse A, Nosarti C, AlAasady M, Stewart A. Cognitive and motor function and the size of the cerebellum in adolescents born very preterm. Brain. 2001;124:60-6.

12. Fallang B, Sauigstad O, Grogaard J, Hadders-Algra M. Kinematic quality of reaching movements in preterm children. Pediatr Res. 2003;53:836-42.

13. Foreman N, Fielder A, Minshell C. Visual search, perception and visual-motor skill in healthy children born at 27-32 week's gestation. J Exp Child Psychol. 1997;64:27-41.

14. Hall A, McLeod A, Counsell C, Thomson L, Mutch L. School attainment, cognitive ability and motor function in a total Scottish very-lowbirth weight population at eight years: a controlled study. Dev Med Child Neurol.1995;37:1037-50. 
15. Jongmans M, Mercuri E, Henderson S, deVries L, Sonksen P, Dubowirz L. Visual function of prematurely born children with and without perceptualmotor difficulties. Early Hum Dev. 1997;45:7382.

16. Liebhart G, Sontheimer D, Linderkamp O. Visualmotor function of very low birth weight and fullterm children at $3 \frac{1}{2}$ to 4 years of age. Early Hum Dev. 2000;57:33-47.

17. Howard K, Roberts G, Lim J, Lee KJ, Barre N, Treyvaud K, et al. Biological and environmental factors as predictors of language skills in very preterm children at 5 years of age. J Dev Behav Pediatr. 2011;32:239-49.

18. Dahl LB, Kaaresen PI, Tunby J, Handegård BH, Kvernmo S, Rønning JA. Emotional, behavioral, social, and academic outcomes in adolescents born with very low birth weight. Pediatrics. 2006;118(2):e449-59.

19. Delobel-Ayoub M, Kaminski M, Marret S, BurguetA, Marchand L, N'Guyen S, et al. Behavioral outcome at 3 years of age in very preterm infants: The EPIPAGE study. Pediatrics. 2006;117:19952005.

20. Vohr BR, Wright LL, Mele L, Verter J, Steichen JJ, Simon NP, et al. Neurodevelopmental and functional outcomes of extremely low birth weight infants in the National Institute of Child Health and Human Development Neonatal Research Network, 1993-1994. Pediatrics. 2000;105:1216-26.

21. Fily A, PierratV, Delporte V, BreartG, Truffert P. Factors associated with neurodevelopmental outcome at 2 years after very preterm birth: the population-based Nord-Pas-de-Calais EPIPAGE cohort. Pediatrics. 2006;117:357-66.

22. Van-Noort-van der Spek, IL, Franken, MCh, Weisglas-Kuperus N. Language functions in pretermborn children: A Systematic review and Meta-analysis. Pediatrics. 2012;129:745-54.

23. Domellof E, Johansson AM, Rönnqvist L. Handedness in preterm born children: A systematic review and a meta-analysis. Neuropsychologia. 2011;49:2299-310.

24. Burnett AC, Anderson PJ, Cheong J, Doyle LW, Davey CG, Wood SJ. Prevalence of psychiatric diagnoses in preterm and full-term children, adolescents and young adults: a meta-analysis. Psychol Med. 2011;41:2463-74.

25. Prachi E, Clements M, Pehlmann J. Maternal resolution of grief after preterm birth: implica- tions for infant attachment security. Pediatrics. 2010;127:284-92.

26. Zwicker JG, Harris SR. Quality of life of formerly preterm and very low birth weight infants from preschool age to adulthood: a systematic review. Pediatrics. 2012;121:366-76.

27. Bhutta AT, Cleves MA, Casey PH, Cradock MM, Anand JS. Cognitive and behavioral outcomes of school-aged children who were born preterm. A meta-analysis. JAMA. 2002;288:728-37.

28. Glennis J, Richardson S. Quality of life of formerly preterm and very low birth weight infants from preschool age to adulthood: a systematic review. Pediatrics. 2008;121(2):366-76.

29. Fernández-Ríos L, Buela-Casal G. Standars for the preparation and writing of Psychology review articles. Int Journal Clin Health Psych. 2009;9:32944.

30. Moher D, Liberati A, Tetzlaff J, Altman DG, The PRISMA Group. Preferred reporting items for systematic reviews and meta-analyses: the Prisma statement. PLoS Med. 2009;6(6):e1000097.

31. Caravale B, Tozzi C, Albino G, Vicari S. Cognitive development in low risk preterm infants at 3-4 years of life. Arch Dis Child Fetal Neonatal Ed. 2005;90:474-79.

32. Hoff Esbjørn B, Hansen BM, Greisen G, Mortensen EL. Intellectual development in a Danish cohort of prematurely born preschool children: specific or general difficulties? J Dev Behav Pediatr. 2006;27(6):477-84.

33. Delobel-Ayoub M, Arnaud C, White-Koning M, Casper Ch, Pierrat V, Garel M, et al. Behavioral problems and cognitive performance at 5 years of age after very preterm birth: The EPIPAGE study. Pediatrics. 2009;123:1484-92.

34. Rose SA, Feldman JF, Jankowski JJ. Infant visual recognition memory: independent contributions of speed and attention. Dev Psychol. 2003;39(3):56371.

35. Hugues MB, Shults J, McGrath J, Medoff-Cooper B. Temperament characteristics of premature infants in the first year of life. J Dev Behav Pediatr. 2002;23:430-53.

36. Feldman R. The development of regulatory functions from birth to 5 years: insights from premature infants. Child Dev. 2009;80:544-61.

37. Evrard D, Charollais A, MarretS, Radi S, Rezrazi P, Mellier D. Cognitive and emotional regulation 
developmental issues in preterm infants 12 and 14 months after birth. Eur J Dev Psychol. 2011;8:17184.

38. Hunnius S, Geuze RH, Zweens MJ, Bos AF. Effects of preterm experience on the developing visual system: A longitudinal study of shifts of attention and gaze in early infancy. Dev Neuropsychol. 2008;33:521-35.

39. Landry SH, Denson SE, Swank PR. Effects of medical risk and socioeconomic status on the rate of change in cognitive and social development for low birth weight children, J Clin Exp Neuropsychol. 1998;1(9):261-74.

40. Pitchford N, Johnson S, Scerif G, Marlow N. Early indications of delayed cognitive development in preschool children born very preterm: Evidence from domain-general and domain-specific tasks. Inf Child Dev. 2011;20:400-22.

41. Espy KA, Senn TE, Charak DA, Tyler J, Wiebe SA. Perinatal $\mathrm{pH}$ and neuropsychological outcomes at age 3 years in children born preterm: An exploratory study. Dev Neuropsychol. 2007;32:669-82.

42. Baron IS, Erickson K, Ahronovich MD, Litman FR, Brandt J. Spatial location memory discriminates children born at extremely low birth weight and late-preterm at age three. Neuropsychology. 2010;24;787-94.

43. Vicari S, Caravale B, Carlesimo GA, Casadei AM, Allemand F. Spatial working memory deficits in children at ages 3-4 who were low birth weight, preterm infants. Neuropsychology. 2004:18:673-8.

44. Clark CC, Woodward LJ, Horwood LJ, Moor S. Development of emotional and behavioral regulation in children born extremely preterm and very preterm: biological and social influences. Child Dev. 2008;79:1444-62.

45. Dall'oglio AM, Rossiello B, Coletti MF, Bultrini M, De Marchis C, Rava L, et al. Do healthy preterm children need neuropsychological follow-up? Preschool outcomes compared with term peers. Dev Med Child Neurol. 2010;52:955-61.

46. Woodward LJ, Clark CAC, Pritchard VE, Anderson PJ, Inder T E. Neonatal white Matter abnormalities predict global executive function impairment in children born very preterm. Dev Neuropsychol. 2011;36:22-41.

47. Briscoe J, Gathercole SE, Marlow N. Everyday memory and cognitive ability in children born very prematurely. J Child Psychol Psychiat. 2001;42:749-54.
48. Roberts G, Lim J, Doyle L, Anderson P. High rates of school readiness difficulties at 5 years of age in very preterm infants compared with term controls. J Dev Behav Pediatr. 2011;32:117-23.

49. Perricone G, Morales R. The temperament of preterm infant in preschool age. Ital J Pediatr. 2011;37:1-7.

50. Sondaar M, van Kesse BJ, de Kleine MJK, Briët JMA, den Ouden L, van Baar A. Do pediatricians recognize cognitive developmental problems in preterm children at age 5 years? Journal Dev Phy Dis. 2008;20:21-9.

51. Nordhov SM, Rønning JA, Dahl LB, Ulvund SE, Tunby J, Kaaresen PI. Early intervention improves cognitive outcomes for preterm infants: Randomized controlled trial. Pediatrics. 2010;126:108894.

52. Cornelieke SH, Aarnoudse-Moens DP, Oosterlaan J, Duivenvoorden HJ, Weisglas-Kuperus N. Executive function in very preterm children at early school age. J Abnorm Child Psychol. 2009;37:98193.

53. Therion JM, Worwa CT, Mattia FR, DeRegnier O. Altered pathways for auditory discrimination and recognition memory in preterm infants. Dev Med Child Neurol. 2004;46:816-24.

54. Cattani A, Bonifacio S, Ferzt M, Iverson JM, Zocconi E, Caselli MC. Communicative and linguistic development in preterm children: a longitudinal study from 12 to 24 months. Int J Lang Commun Disord. 2010;45:162-73.

55. Jansson-Verkasalo E, Valkama M, Vainionpaa L, IIlko E, Lehtihalmes M. Language development in very low birth weight preterm children: a follow-up study. Folia Phoniatr Logop. 2004b;56:108-19.

56. Kern S, Gayraud F. Influence of preterm birth on early lexical and grammatical acquisition. First Lang. 2011;27:159-73.

57. Foster-Cohen S, Edgin JO, Champion PR, Woodward LJ. Early delayed language development in very preterm infants: evidence from the MacArthur-Bates CDI. J Child Lang. 2007;34:655-75.

58. Marston L, Galvert S, Grenough A, Marlow N. Factors affecting vocabulary acquisition at age 2 in children born between 23 and 28 week gestation. Dev Med Child Neurol. 2007;49:591-6.

59. Stolt S, Klippi A, Launonen K. Size and composition of the lexicon in prematurely born very low birth weight and full term Finnish children at two years of age. J Child Lang. 2007;34:283-10. 
60. Briscoe J, Gathercole SE, Marlow N. ShortTerm Memory and Language outcomes after extreme prematurity at birth. J Speech Lang Hear Res.1998;41:654-66.

61. Van Lierde KM, Roeyers H, Boerjan, S, Groote, I. Expressive and receptive language characteristics in three year old preterm children with extremely low birth weight. Folia Phoniatr Logop. 2009;61:2969.

62. Jansson-Verkasalo E, Korpilathi P, Jantti V, Valkama M, Vainionpää L, Alku P, et al. Neurophysioloic correlates of déficit phonological representations and object naming in premeturely born children. Clin Neurophysiol. 2004;115:179-87.

63. Luoma L, Herrgard E, Martikainen A,Ahonen T. Speech and language development of children born at $<32$ week'gestation: a 5-years prospective followup study. Dev Med Child Neurol. 1998;40;380-7.

64. Wolke D, Meyer R. Cognitive status, language attainment, and prereading skills of 6 years old very preterm children and their peers: the Baravian longitudinal study. Dev Med Child Neurol. 1999;41:94-109.

65. Wolke D, Samara M, Bracewell M, Marlow N. Specific language difficulties and school achievement in children born at 25 weeks of gestation or less. J Pediatr. 2008;152:256-62.

66. Cevasco AM. The effects of mothers'singing on full-term and preterm infants and maternal emotional responses. J Music Ther. 2008;45:273-306.

67. Tooten A, Hoffenkamp H, Hall R, Winkel FW, Eliëns M, Vingerhoets A, et al. The effectiveness of video interaction guidance in parents of premature infants: A multicenter randomized controlled trial. BMC Pediatrics. 2012;12:76.

68. Ravn IH, Smith L, Smeby N, Kynoe N, Sandvik L, Bunch EH, et al. Effects of early motherinfant intervention on outcomes in mothers and moderately and late preterm infants at age 1 year: A randomized controlled trial. Infant Behav Dev. 2012;35:36-47.

69. Gray PH, Edwards DM, O'Callaghan MJ, Cuskelly M. Parenting stress in mother of preterm infants during early infancy. Early Hum Dev. 2012;88:459.

70. Spittle A, Treyvaud K, Doyle L, Roberts G, Lee $\mathrm{K}$, Inder T, et al. Early emergency of behavior and social-emotional problems in very preterm infants. J Am Acad Child Adolesc Psychiatry. 2009;48:90918.

71. Treyvaud K, Anderson VA, Howard K, Bear M, Hunt RW, Doyle L, et al. Parenting behavior is associated with the early neurobehavioral development of very preterm children. Pediatrics. 2009;123:555-61.

72. Treyvaud K, Anderson VA, Lee KJ, Woodward L, Newnham C, Inder TE, et al. Parental mental health and early social-development of children born very preterm. J Pediatr Psychol. 2010;35:76877.

73. Beckwith L, Rodning C. Dyadic processes between mothers and preterm infants: Development at ages 2 to 5 years. Infant Mental Health Journal. 1996;17(4);322-33.

74. Assel MA, Landry SH, Swank PR, Steelman L, Miller-Loncar C, Smith KE. How do mother's childrearing histories, stress and parenting affect children's behavioral outcomes? Child Care Health Dev. 2002;28(5):359-68.

75. Landry SH, Smith KE, Swank PR, Miller-Loncar CL. Early maternal and child influences on children's later independent cognitive and social functioning. Child Dev. 2000;71:358-75.

76. Lavta R. How is maternal recollection of the birth experience related to the behavioral and emotional outcome of preterm infants? Early Human Dev and Biotech Week. 2008;12:205-81.

77. Saavalainen P, Luoma L, Bowler D, Määttä S, Kiviniemi $\mathrm{V}$, Laukkanen $\mathrm{E}$, et al. Spatial span in very prematurely born adolescents. Dev Neuropsychol. 2007;32(3):769-85.

78. Johnson S. Cognitive and behavioural outcoms following very preterm birth. Semin Fetal Neonatal Med. 2007;12(5):363-73.

79. Marlow N, Wolke D, Bracewell MA, Samara M. Neurologic and developmental disability at six years of age after extremely preterm birth. NEJM. 2005;352(1):9-19.

80. Illay G, Umran O, KiIzilkaya N. Biopsychosocial risk factors for preterm birth and postpartum emotional well-being: a case-control study on Turkish women without chronic illnesses. J Clin Nurs. 2011;20:653-65. 